\title{
Lipid Droplet metabolism dependent microbial defense in pre-immune zebrafish embryos
}

\author{
Asmita Dutta, Sampali Banerjee and Deepak Kumar Sinha*
}

Running title: LD-mediated pre-immune defense of zebrafish embryos

Keywords: zebrafish embryos, LDs, Lipid Droplet Density, lipolysis, microbial defense

\begin{abstract}
Microbes present survival challenge to pre-immune embryos. Our study provides evidence for antimicrobial-secretion-based strategy of zebrafish embryos against microbes during pre-immune stages. Chorion prevents physical contact between embryos and microbes, yet microbes compromise embryonic survival through their secretions. Development of embryos in microbe-free medium involves secretion of pro-microbial compounds that bacteria utilize to accelarete growth. Embryo senses presence of microbes through microbial secretions. They respond by altering their secretions to include antimicrobial compounds along with regular promicrobial ones. Upon sensing embryonic anti-microbial secretions, microbes too alter their secretions to include more potent toxins for embryos. In response to this embryos alter their secretions to include more potent antimicrobial compounds. Ability of embryos to secrete antimicrobial compounds is positively correlated with amount of lipid droplets (LDs) in them. Inhibition of LD metabolism prevents antimicrobial secretions by embryos. Thus, LDs protect zebrafish embryos from microbes. This manuscript establishes that pre-immune embryos employ dynamically evolving biochemical warfare to protect themselves from harmful microbes.
\end{abstract}

\section{Introduction}

The immune systems of fish and mammals share significant similarities. For example, both the vertebrates host comparable sets of lymphocytes (similar T-cell subsets) and hence, generate analogous immune responses ${ }^{1-3}$. However, unlike mammalian embryos that develop inside the body of the female, embryonic development of fish (oviparous) occurs in the open aquatic environment. Therefore, right after fertilization these oviparous embryos require a functional immune system to safeguard them from pathogenic microorganisms present in their natural habitat. Studies on zebrafish embryos report that adaptive immune response develops four to six weeks after fertilization of the $\operatorname{egg}^{4-6}$. Further, 'primitive macrophages' appear only after 16 hours post fertilization (hpf ${ }^{7-9}$. These macrophages and the newly generated neutrophils constitute the innate immune system in one day old zebrafish embryos ${ }^{2,10}$. Hence, these embryos are exposed 
32 to a large number of pathogens prior to the development of either the innate or the adaptive

33 immune system. However, it is not known how the zebrafish embryos protect themselves from

34 pathogens before 1 day post fertilization (dpf) when even the innate immune system is not

35 functional. Lipid droplets (LDs) are fat reservoirs with a neutral lipid core delimited by a

36 phospholipid monolayer studded with various proteins ${ }^{11-13}$. Recent investigations have

37 recognized the importance of LDs beyond lipid metabolism and homeostasis. They exhibit other

38 non-canonical functions ${ }^{14-16}$ in addition to coordination of immune responses ${ }^{17}$. The lipid and the

39 protein content of the LDs determine the inflammatory activity of the myeloid cells, thereby

40 suggesting a crucial role in determining the cellular functionalities ${ }^{18,19}$. LDs in Drosophila

41 embryos are reported to host anti-bacterial properties. In addition to LDs, the antimicrobial

42 phosvitin $(\mathrm{Pv})$, a nutritional protein abundant in eggs, protects the embryos from microbes. ${ }^{20}$

43 In zebrafish embryos, the chorion prevents all physical contact between the embryo body and the

44 microbes thus the antimicrobial properties of LDs or Pv are not useful in dealing with the extra-

45 embryonic microbes. The pathogenic microbes in the aqueous environment however challenge

46 the embryos by releasing secretions which are permeable to the chorion. Strategies by the

47 embryos to counter pathogenic challenges remain unknown. In this manuscript we explore how

48 the embryos oppose the pathogens during early development (i.e. until 9 hpf). Our results

49 indicate that LD metabolism is central to the embryonic strategies (until $9 \mathrm{hpf}$ ) that mitigate

50 challenges posed by pathogenic microbes in aquatic environment.

\section{Results}


(A)

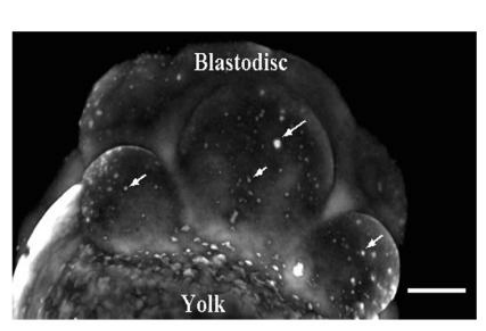

(B)

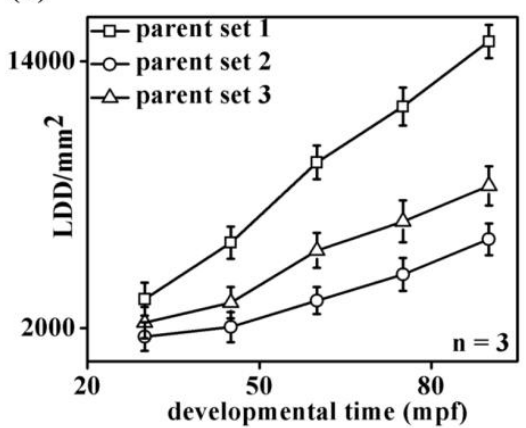

(C)

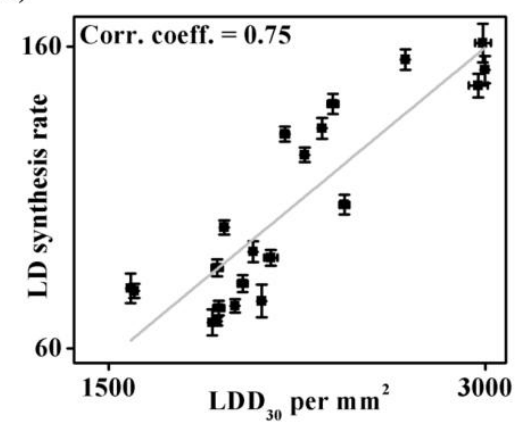

(D)

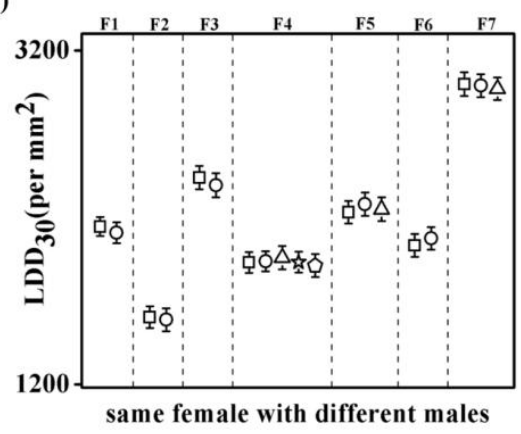

(E)

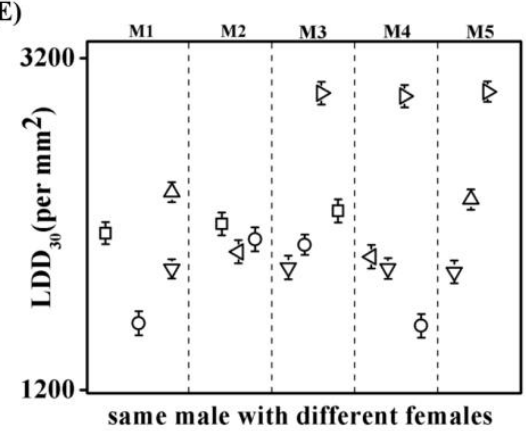

Figure 1. Embryonic LDD is a maternal characteristic. (A) Fluorescence image of 8-cell stage embryo stained with LD540, arrows show LDs, scale bar $100 \mu \mathrm{m}$. (B) Average LDD for a minimum of five embryos each, from three representative parent sets plotted against developmental time (mpf). Error bars are obtained from embryos acquired across different clutches from the same parents $(n=3)$. (C) LD synthesis rate (rate-of-increase of LDD) plotted as a function of respective embryonic $\mathrm{LDD}_{30}$ values. Fit line denotes a positive correlation (0.75). (D) $\mathrm{LDD}_{30}$ re-plotted for 7 independent female parents (F1 to F7) bred with different males (different symbols). (E) $\mathrm{LDD}_{30}$ re-plotted for 5 independent male parents (M1 to M5) bred with different females (different symbols), error bars denote Standard Error of Mean (S.E.M.).

\section{Mother zebrafish alone dictates the embryonic LD density (LDD)}

The zebrafish embryos contain large number of LDs distributed cortically in the blastodisc (Fig.

1A). Fig. 1B compares the density of LDs (the number of $\mathrm{LDs} / \mathrm{mm}^{2}$ of the blastodisc) in the embryos obtained from three different sets of parents. In accordance to our previous report ${ }^{21}$, the LDD increases with the development of the embryos, however, we also observe a unique $\operatorname{LDD}_{30}$ value (LDD at 30mpf) associated with each parent set (Fig. 1B, Supp. S1). Interestingly, the rateof-increase of LDD (slope) with embryonic development, for embryos from a particular parent set, exhibits a strong positive correlation (correlation coefficient $=0.75$ ) with the corresponding 
71 upon the initial LDD values $\left(\mathrm{LDD}_{30}\right)$, which in turn depends upon the respective parent set used

72 for breeding. Next, we explored whether the unique embryonic $\mathrm{LDD}_{30}$ is determined

73 independently or jointly by the male and the female parents. We found that the $\mathrm{LDD}_{30}$ of the

74 embryos obtained from independent females bred with different males do not alter $\operatorname{LDD}_{30}$

75 significantly (Fig. 1D), while similar analysis with the same male fish bred with different

76 females show significantly varying $\mathrm{LDD}_{30}$ values in the offspring (Fig. 1E). Hence, embryonic

77 LDD is uniquely determined by the female parent alone. This allowed us to obtain the embryos

78 with distinct LDD by selecting the appropriate females for breeding.

(A)

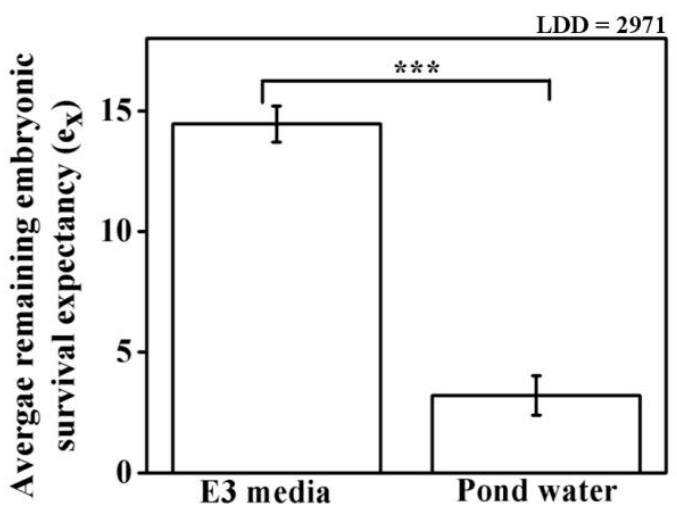

(C)

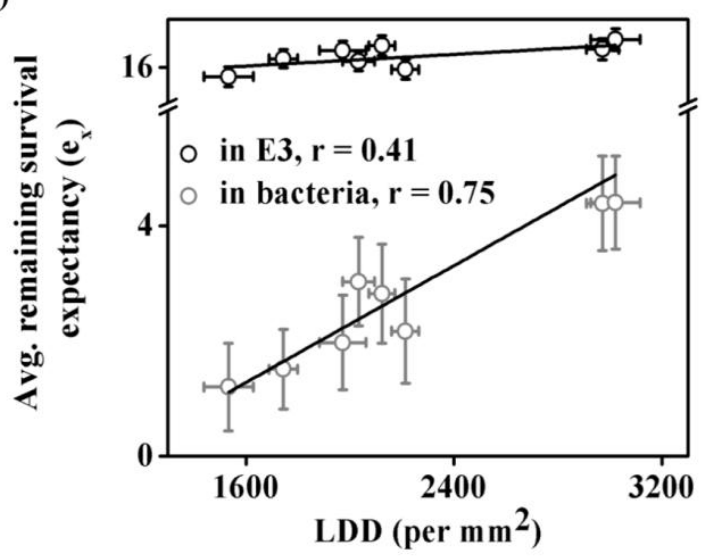

(B)

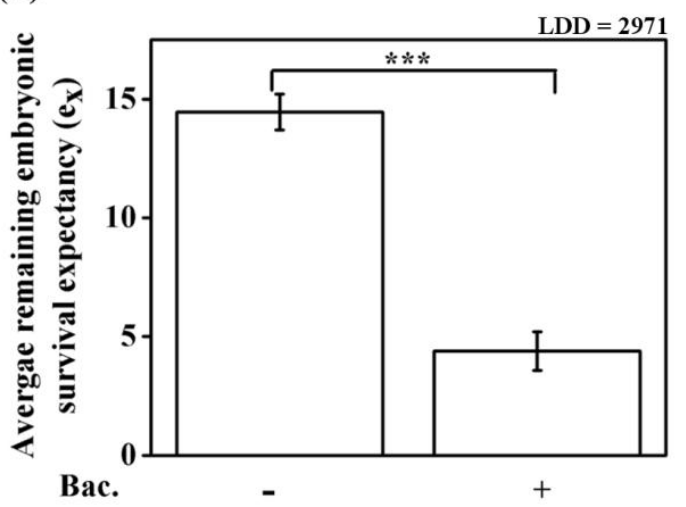

(D)

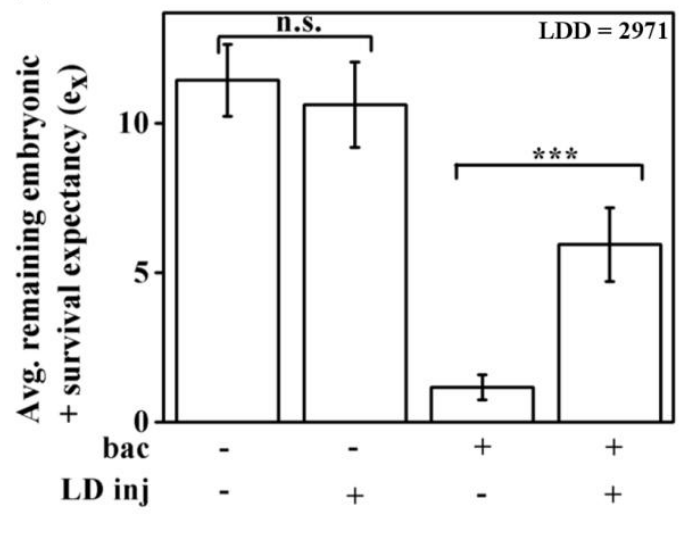

Figure 2. Survival of embryos in presence of microbes is dependent upon LDD. (A) Average 
mentioned in x-axis). (B) $\mathrm{e}_{\mathrm{x}}$ of embryos incubated in the presence (left bar) or absence of bacteria (right bar) (as menitoned in $\mathrm{x}$-axis). (C) $\mathrm{e}_{\mathrm{x}}$ of embyros kept in E3 containing bacteria (gray) or in $\mathrm{E} 3$ alone (black) plotted against corresponding $\mathrm{LDD}_{30}$ values. Correlation fit shows a Pearson coefficient of 0.75 (with bacteria in E3) and 0.48 (in E3). (D) $e_{x}$ of embryos incubated with/without bacteria in E3, with/without exogenous LD injection. $\mathrm{LDD}_{30}$ of the embryo clutch is $2971 / \mathrm{mm}^{2}$ in A, B, D. Bacterial concentration used: $10^{6} / \mathrm{ml}$. All embryos incubated with external food supply so that bacteria is the only challenge to embryos.

\section{LDs help the embryos endure microbial challenges}

In the natural habitats, the zebrafish embryos are exposed to different microbes prevalent in the aqueous environment. These microbes do challenge survival of the embryos. Life expectancy $\left(e_{x}\right)$ of embryos is remarkably lower in water obtained from local pond (natural habitat) compared to sterile E3 (media prepared in laboratory) (Fig. 2A). To understand the impact of microbes on the survival of embryos, we incubated the embryos in bacteria-laced E3 media. For embryos with a given LDD, presence of bacteria (gram negative/gram positive) in E3 reduces their $\mathrm{e}_{\mathrm{x}}$ in a concentration dependent manner (Supp. S2). We selected a bacterial concentration of $10^{6} / \mathrm{ml}$ at which the survival chances of embryos (LDD=2971) diminishes to $50 \%$ at $24 \mathrm{hpf}$ (Supp. S2). We observe a similar reduction in likelihood of survival of embryos in E3 laced with bacteria (Fig. 2B). Interestingly, the embryos exhibit $\mathrm{LDD}_{30}$ dependent life expectancy in the presence of microbes (Supp. S3), as confirmed by the corresponding higher correlation coefficient ( $\mathrm{r}=0.75$ in presence of microbes) in Fig. $2 \mathrm{C}$. Therefore, we hypothesize that the LDs are components of the antimicrobial defense system of the nascent zebrafish embryos. This hypothesis is further validated by injecting additional LDs into newly laid embryos and incubating them with/without bacteria. Exogenous LDs in the embryos increases their survival in bacteria-laced medium (Fig. 2D). However, exogenous LDs have no impact on the embryonic survival in bacteria-free medium (i.e. in E3) (Supp. S4). Hence, the LDs assist the embryos to counter the pathogenic challenge presented by the microbes. 
(A)

(i)

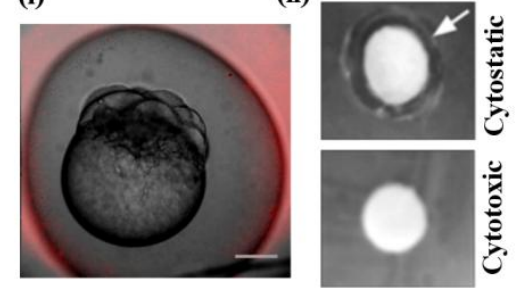

(B)

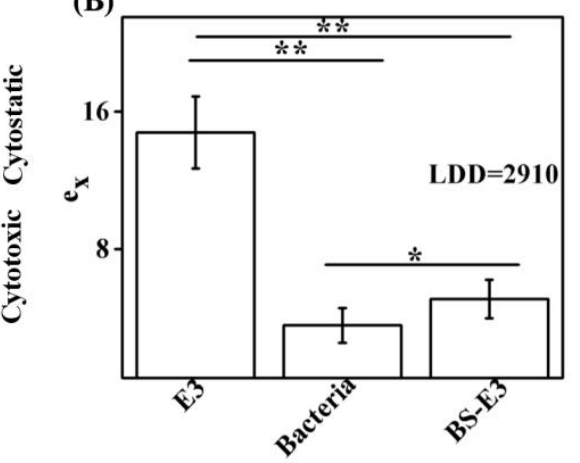

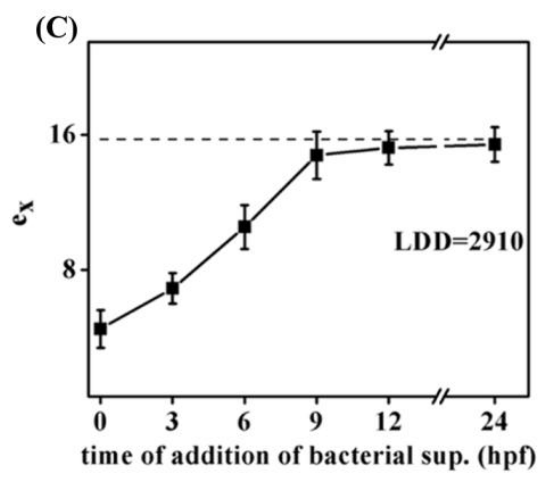

(D)

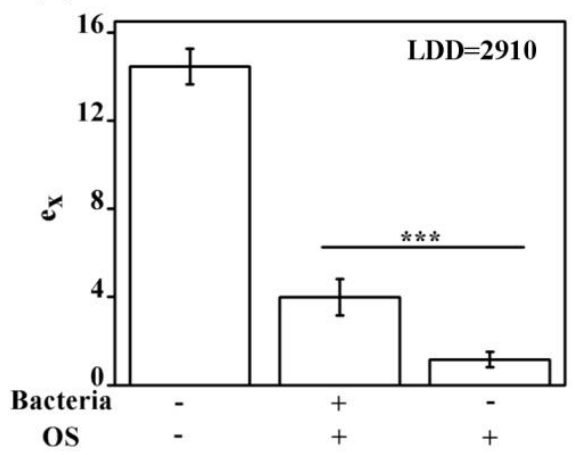

(E)

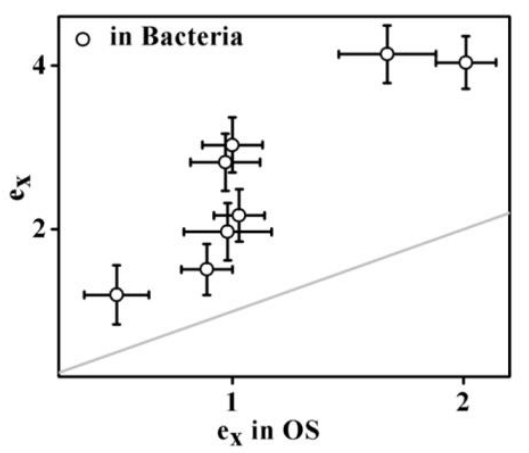

Figure 3. Embryonic survival under bacterial exposure is dependent upon LD-lipolysis. (A) (A) (i) Over-laid image of embyro (DIC) and RFP-E.coli (fluorescence) showing the inability of the bacteria to penetrate the chorion, scale bar $300 \mu \mathrm{m}$. (ii) Disc diffusion assay for cytostatic (upper) and cytotoxic (lower) property assesment of chorion against E.coli. Arrow: zone of inhibition. (B) $\mathrm{e}_{\mathrm{x}}$ for embryos incubated in E3 (left bar), with bacteria in E3 (middle bar), in BSE3 (right bar). (C) $e_{x}$ for embryos incubated in bacterial supernatant added at different durations (x-axis denotes different time points when BS-E3 was added to embryos, in terms of hpf). Dash line denotes $e_{x}$ of control embryos (grown in E3). (D) Plot of $e_{x}$ verus the different conditons of incubation. The embryos were incubated in the presence/absence of bacteria and $+/-$ OS in the medium. The embryo clutch used has LDD $2910 / \mathrm{mm}^{2}$. (E) $\mathrm{e}_{\mathrm{x}}$ of embryos plotted versus $\mathrm{e}_{\mathrm{x}}$ in OS containing E3 for embryos incubated in bacteria (black). Different data point indicate embryos with different $\mathrm{LDD}_{30}$ values. Gray line denotes straight line $(\mathrm{y}=\mathrm{x})$.

\section{Lipolysis of LDs is necessary to confer defense against microbes}

122 LDs present in the Drosophila embryos are reported to exhibit antimicrobial properties ${ }^{22}$. Our studies too reveal that the LDs isolated from zebrafish embryos host anti-bacterial property in counter the microbial challenges then they must come in physical contact with the microbes. To 
127 and the outer membrane of the embryos. For this the embryos were incubated in RFP-E.coli

128 laced E3. Fig. 3A (i) establishes that the bacteria (in green) fail to penetrate even the outer

129 chorion of the embryo (in gray). Though, the chorion prevents any physical contact between the

130 embryo and the bacteria, yet presence of bacteria in E3 is deleterious to embryonic survival. This

131 therefore, suggests that bacteria release permeable deleterious secretions that cause decrease in

132 embryonic life expectancy. Since the chorion is the first line of defense that comes in physical

133 contact with the microbes, we studied its antimicrobial activity. We find that though the chorion

134 lacks cytotoxic capability, it is cytostatic (Fig. 3A (ii)). However, the antimicrobial capabilities

135 of the chorion are not sufficient to protect the embryos from higher concentration of microbes.

136 Next we investigated the effect of deleterious bacterial secretions (BS-E3: obtained by

137 incubating bacteria in E3 overnight and filtering the supernatant; refer Table 1) on embryonic life

138 expectancy $\left(\mathrm{e}_{\mathrm{x}}\right)$ as a function of embryonic developmental stage.

\begin{tabular}{|l|l|l|}
\hline \multicolumn{1}{|c|}{ Nomenclature } & \multicolumn{1}{|c|}{ Secretion from } & \multicolumn{1}{|c|}{ Incubation medium } \\
\hline ES-E3 & Embryo & $\begin{array}{l}\text { Embryonic secretions in E3 } \\
\text { buffer }\end{array}$ \\
\hline BS-E3 & Bacteria & E3 buffer \\
\hline ES-BS & Embryo & $\begin{array}{l}\text { (BS-E3): Bacterial secretions } \\
\text { in E3 }\end{array}$ \\
\hline ES-BS $\mathrm{ES}$ & Embryo & $\begin{array}{l}\text { (BS-ES): Bacteria secretions } \\
\text { in ES-E3 }\end{array}$ \\
\hline ES-BS(OS) & Embryo & $\begin{array}{l}\text { (BS-E3 + 10 } \mu \text { M OS): } \\
\text { Bacterial secretions in E3 with } \\
\text { OS }\end{array}$ \\
\hline BS-ES & $\begin{array}{l}\text { ES-E3 Embryonic secretions } \\
\text { in E3 }\end{array}$ \\
\hline BS-ES BS & Bacteria & $\begin{array}{l}\text { ES-BS: Embryonic secretions } \\
\text { in BS-E3 }\end{array}$ \\
\hline
\end{tabular}

139 Table 1. Nomenclature for different media obtained from secretions under different conditions.

140 Similar to incubation in bacteria-laced-E3, embryonic incubation in BS-E3 (a solution free of

141 bacteria but contains bacterial secretions) also leads to significant decrease in the survival of the 
142 embryos (Fig. 3B). Embryos exposed to BS-E3 right from 0 hpf (1 cell stage) exhibit lower life 143 expectancy as compared to the embryos exposed to BS-E3 at later developmental stages (i.e 9

144 hpf onwards, Fig. 3C). Since higher LDD bestows embryos with better capabilities to counter 145 microbial challenge during 0-9 hpf (Fig. 2C), hence we investigated if metabolism of the LDs is 146 required for such defense capabilities against microbes. The breakdown of LDs by the cytosolic 147 lipases is one of the primary pathways for the catabolism of lipids and subsequent energy 148 generation $^{12}$. To investigate the role of LD metabolism in the survival of the embryos under 149 bacterial invasion, we used the broad spectrum lipolysis inhibitor, Orlistat (OS) ${ }^{23,24}$, to reduce the 150 activity of the embryonic lipases. Treatment of the embryos with OS at the working 151 concentration severely retards the development of the zebrafish embryos ${ }^{25}$. Therefore, we titrated 152 the concentration of OS to determine the appropriate concentration $(10 \mu \mathrm{M})$ that does not affect 153 the rate of development of the embryos significantly (Supp. S6). Irrespective of the LDD, 154 inhibition of LD lipolysis by OS reduces embryonic life expectancy in bacteria-laced-E3 (Fig. $1553 \mathrm{D}, 3 \mathrm{E})$. Thus, metabolism of $\mathrm{LDs}$ is an integral part of the embryonic strategy to combat 156 deleterious bacterial secretions, and mere presence of LDs is not sufficient to bestow embryos 157 with microbe countering capabilities. 
(A)

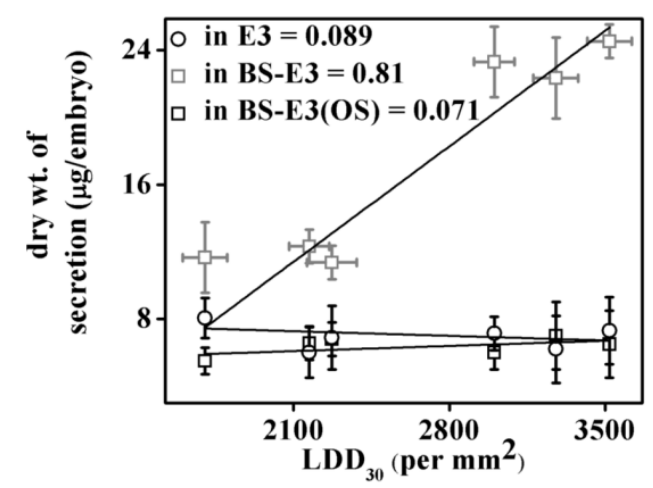

(C)

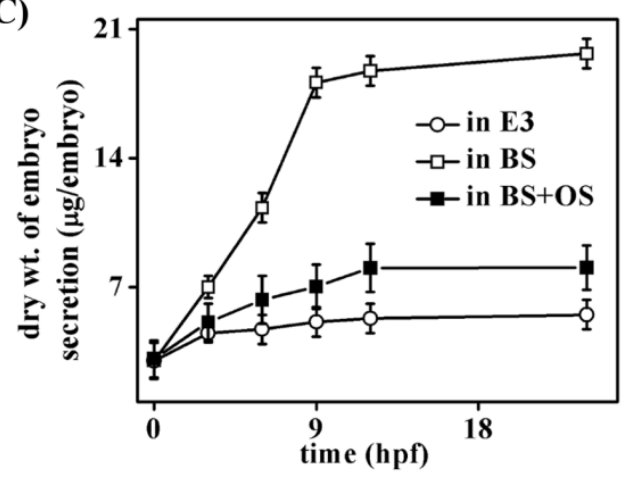

(F)

(i)

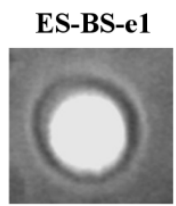

(ii)

(D)

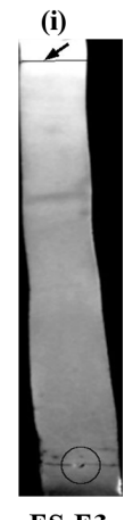

ES-E3
(B) (i) $_{\text {}}$
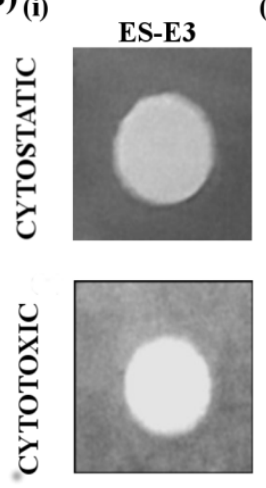

(ii)
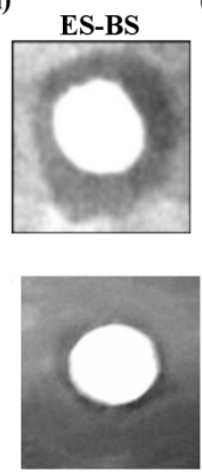

(E)

(iii)
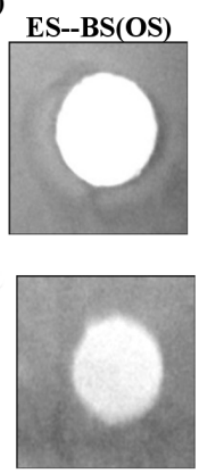

(ii)

(G)

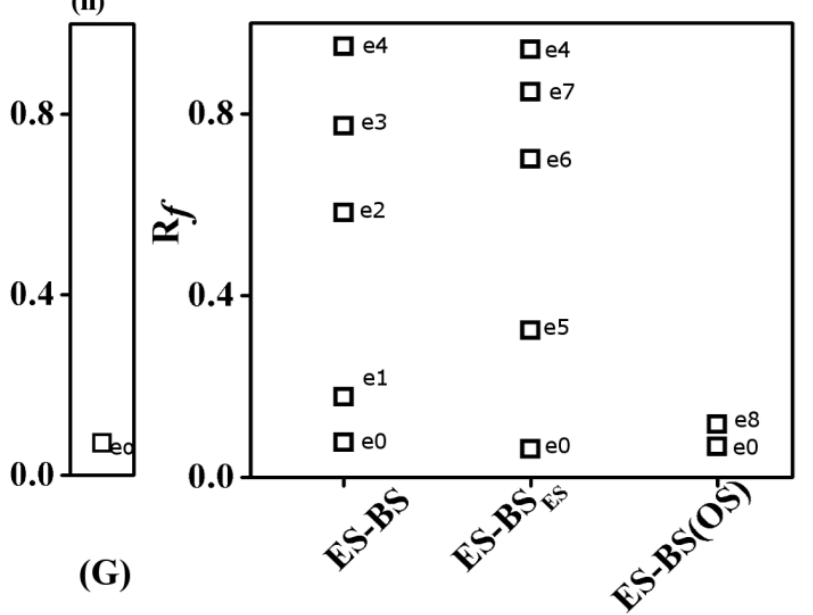

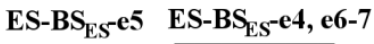

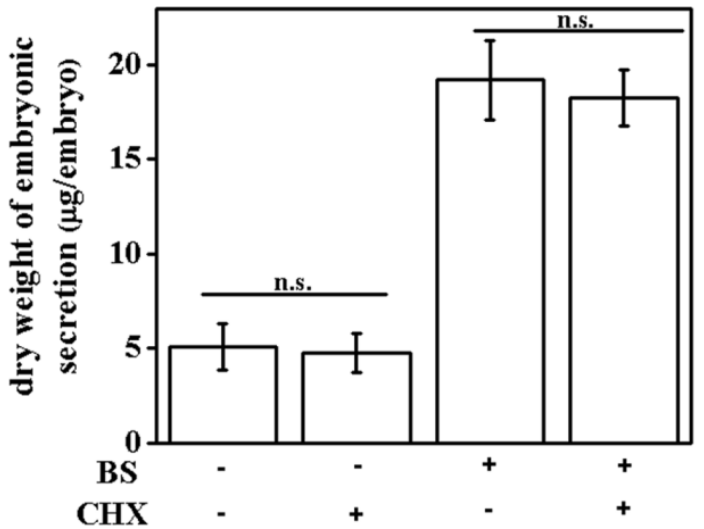

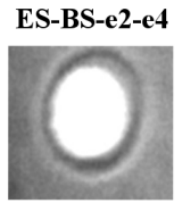

(iii)
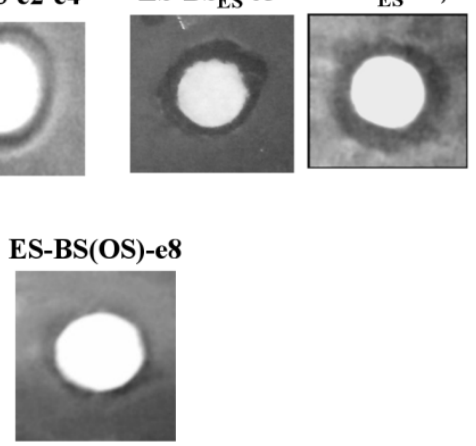

Figure 4. Lipolysis dependent secretion is released by embryos when incubated in BS-E3. (A) Dry weight of secretion ( $\mu \mathrm{g} / \mathrm{embryo})$ of components when embryos are incubated in E3 (circle), BS-E3 (gray, square) and OS $(10 \mu \mathrm{M})$ added to BS-E3 (square, black) plotted against respective $\mathrm{LDD}_{30}$, correlation coefficient provided as legend. (B) Disc diffusion assay: cytostatic (upper panel) and cytotoxic (lower panel) property assesment of (i) ES-E3, (ii) ES-BS, (iii) ES$\mathrm{BS}(\mathrm{OS})$. (C) Cumulative dry weight of embryonic secretion of components ( $\mu \mathrm{g} / \mathrm{embryo}$ ) incubated in E3 (circle), BS-E3 (open, square) and OS $(10 \mu \mathrm{M})$ containing BS-E3 (closed, square). Error bars denote S.E.M. (D) (i) TLC profile of ES-E3 stained by Ninhydrin. Arrow 
shows the solvent front, (ii) Corresponding $\mathrm{R}_{f}$ values for each spot on TLC plate plotted against the respective embryo incubation conditons. (E) Plot of $\mathrm{R}_{f}$ of TLC spots stained with Ninhydrin for embryonic secretions obtained from different incubation conditions as mentioned in $\mathrm{X}$-axis.

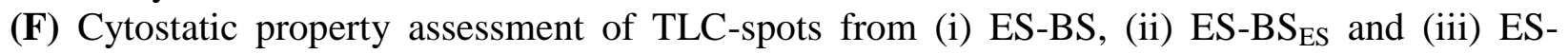
$\mathrm{BS}(\mathrm{OS})$ (numbering as per E). (G) Dry weight of secretion ( $\mu \mathrm{g} / \mathrm{embryo}$ ) for embryos incubated with/without BS-E3 supplememted with/without CHX.

\section{Embryonic antimicrobial secretions mitigate the microbes challenge}

Fig. 4A depicts the level of secretion obtained from embryos with distinct $\operatorname{LDD}_{30}$ values kept under different incubation conditions. We find that the level of embryonic secretions in E3 (ESE3) is independent of LDD ( $\mathrm{r}=0.089)$. However, incubation of embryos in BS-E3 leads to increase in the level of secretions for all the embryos (with different $\mathrm{LDD}_{30}$ values from different female parents) (Fig. 4A). As expected, additional embryonic secretion (ES-BS) is dependent on lipolysis of the LDs and inhibition of lipolysis by OS reduces the levels of embryonic secretions in BS-E3 incubation media (Fig. 4A). Thus, the embryos release lipolysis dependent additional secretions to counter the challenges presented by BS-E3. We had observed earlier that the survival of the embryos in bacteria is LDD dependent (Fig. 3C). This is consistent with the observation that the embryos release higher levels of secretions in BS-E3 if the embryonic secretions host antimicrobial property. Fig. 4B depicts the antimicrobial activity of embryonic secretions under different conditions. While ES-E3 is neither cytostatic nor cytotoxic (Fig. 4B-i), ES-BS does exhibit antimicrobial (cytostatic) activity (Fig. 4B-ii). As expected, ES-BS obtained from OS containing media does not host any anti-bacterial property (Fig. 4B-iii). Therefore, the embryos release LD-lipolysis dependent cytostatic secretions to mitigate the bacterial challenges in their surroundings. Since zebrafish embryos are susceptible to BS-E3 just until 9hpf (Fig. 3C), we investigated the cumulative embryonic secretions i.e. ES-BS until a certain developmental stage (or time in $9 \mathrm{hpf}$ ). Fig. 4C depicts the cumulative amount of secretions released by the embryos when exposed to bacterial secretions i.e. ES-BS until a given developmental stage. We 
observe that the embryos in E3 secrete mainly during the initial $3 \mathrm{hpf}$. There is no significant secretion 3hpf onwards (Fig. 4C). On the other hand, the embryos exposed to BS-E3 continue to release secretions until 9 hpf at a much higher rate. Interestingly, beyond 9 hpf neither are the embryos vulnerable to BS-E3 (Fig. 3C), nor do they secrete antimicrobial compounds (Fig. 4C). Next, we characterized the embryonic secretions using Thin Layer Chromatography (TLC), which can resolve the components present in the secretions based on their polarity. Fig. 4Di is a representative TLC profile when ES-E3 is run on a TLC plate and stained with Ninhydrin (for amino acid detection). The corresponding values of the resolving fractions $\left(\mathrm{R}_{f}\right)$ for the spots were plotted in Fig. 4Dii. $\mathrm{R}_{f}$ defines the migration of a particular component on the TLC plate w.r.t. the migration of the solvent front. Similarly, the TLC profiles of the embryonic secretions under different conditions (ES-BS, ES-BS $\mathrm{ES}_{\text {) }}$ (Table 1) have been represented in terms of their corresponding $\mathrm{R}_{f}$ values (Fig. $4 \mathrm{E}$ ). The secretions by the embryos (ES-BS) incubated in BS-E3 (Fig. 4E, left panel of scatter plots) has significantly different TLC profile compared to ES-E3 (Fig. 4Dii). This suggests that embryos secrete many new compounds (e1-e7) in response to bacterial toxins (BS-E3). Most interestingly, the TLC profile of embryonic secretions, ES-BS, differ from that of $\mathrm{ES}-\mathrm{BS}_{\mathrm{ES}}$, (Fig. 4E, Suppl. S7), probably due to the different material compositions of BS-E3 and BS-ES. Thus, bacteria too have different secretions in E3 and ES-E3. As expected inhibition of LD lipolysis (by OS treatment) inhibits secretion of all compounds (e1e7), instead we find an additional secretion spot (i.e. e8) that was not seen earlier. TLC plate of same samples in a non-polar solvent exhibit no spot with lipid-charring solution, thereby proving that the embryonic secretions are amine group containing compounds (probably amino acids) and not lipid based compounds. Both the secretions ES-BS and ES-BS $\mathrm{ES}_{\text {host }}$ cytostatic property (Fig. 4Fi, ii). However, inhibition of lipolysis results in the loss of antibacterial property of the 
embryonic secretions (Fig. 4Fiii). Release of amino acid based embryonic secretions upon BS-E3 exposure may be a result of either enhanced protein translation or active protein degradation,

218 both of which are characteristic phenomena of early embryonic development. We determined an

219 appropriate concentration of the protein translation inhibitor, Cycloheximide (CHX), at which

220 the embryos survive however, they do show a reduction in the level of total protein due to $\mathrm{CHX}$

$221(1 \mu \mathrm{M})($ Supp. S8). $1 \mu \mathrm{M}$ of CHX does not result in any significant difference in the level of ES-

222 E3 or ES-BS (Fig. 4G). Similarly, neither the treatment of Heclin (inhibitor of protein

223 degradation) produced any significant difference in the level of ES-E3 or ES-BS (Supp. S9).

224 Surprisingly, neither protein translation nor protein degradation seems to be the mechanism of 225 synthesis of the cytostatic secretions (e1-e7). Next we explored the mechanism by which the microbes challenge the embryos. 
(A)

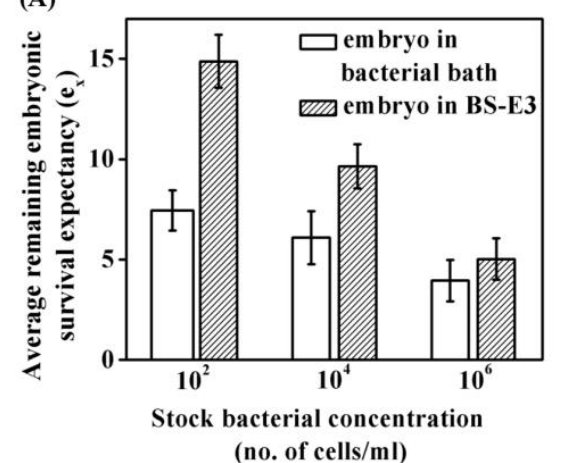

(D)

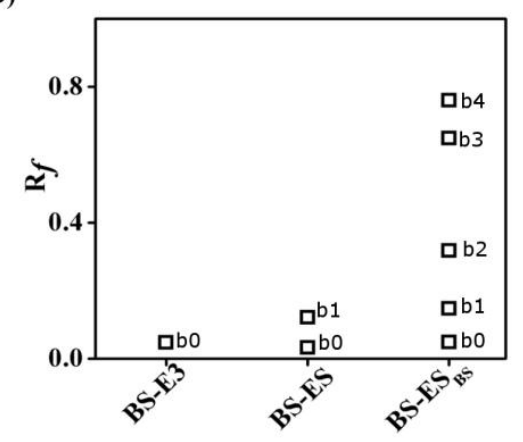

(B)

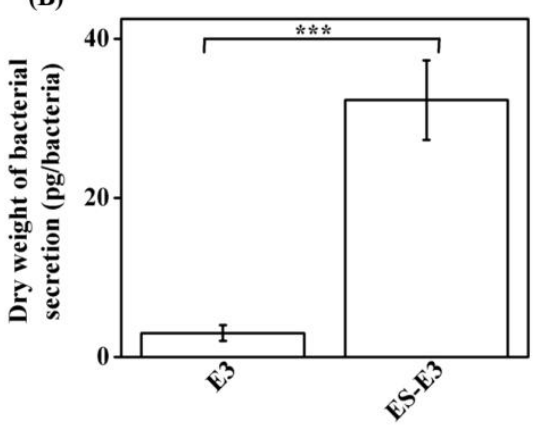

(E)

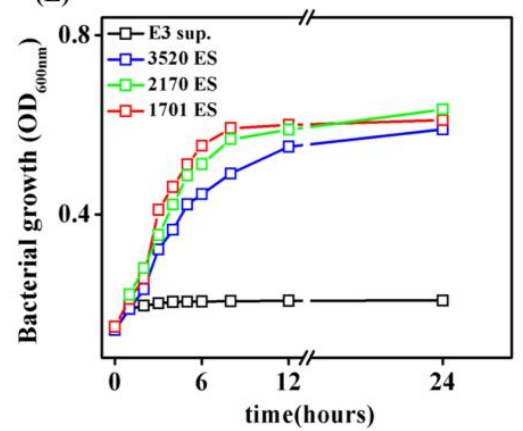

(6)

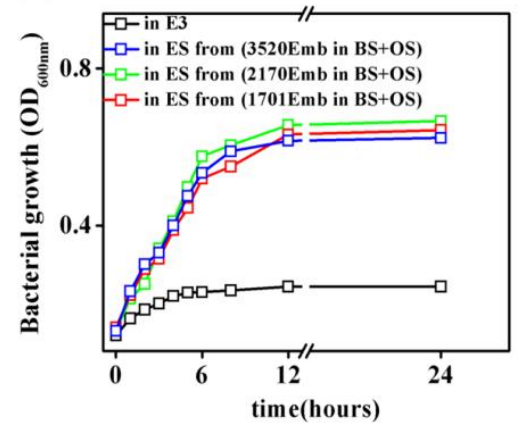

(C)

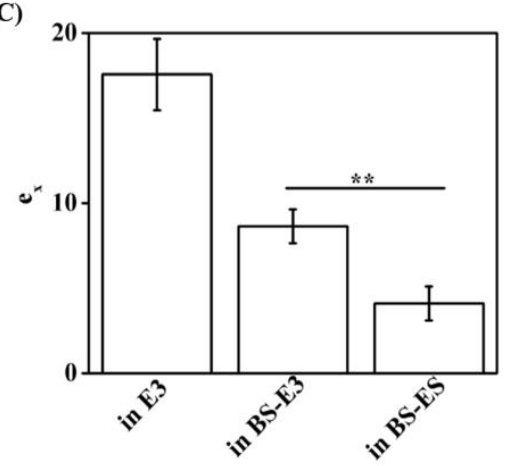

(F)

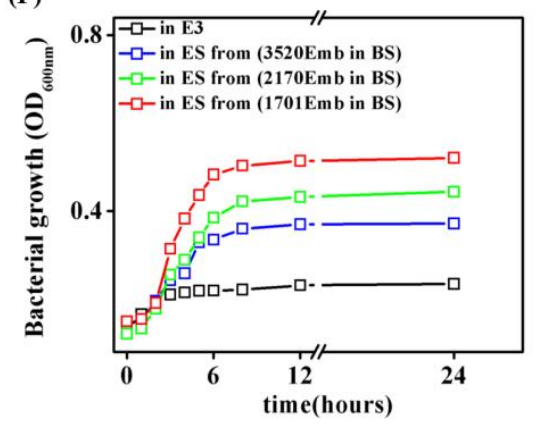

Figure 5. Mechanism of protection of embryos from bacteria by LDs. (A) $e_{x}$ plotted against concentration of the bacterial stock. Embryos were incubated in either bacterial bath (empty bar) or in BS-E3 (filled bar). (B) Dry weight of secretion (pg/bacteria) by bacteria grown in E3 and ES-E3 plotted against the incubation conditions as mentioned in the $\mathrm{x}$-axis. (C) $\mathrm{e}_{\mathrm{x}}$ plotted against different incubation conditions as mentioned in the x-axis. Same amount of secretion (1 $\mathrm{mg} / \mathrm{ml}$ ) was suspended in E3 and then used to treat the embryos. (D) Plot of $\mathrm{R}_{f}$ of spots obtained

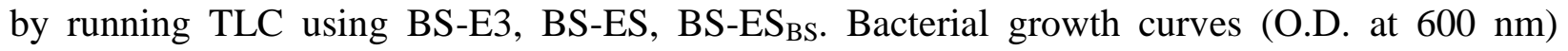
plotted against time (hours) for bacteria grown in (E) ES-E3 obtained from different embryo sets (with different $\mathrm{LDD}_{30}$ ), (F) ES-BS from different embryo sets, (G) ES-BS (OS) obtained from different embryo sets. Graph legends denote the $\mathrm{LDD}_{30}$ values of the embryo clutches. 
We investigated the nature of the bacterial secretions under different incubation conditions. As expected, we observe a concentration dependent effect of BS-E3 on life expectancy $\left(\mathrm{e}_{\mathrm{x}}\right)$ of the embryos (Fig. 5A). The secretion, BS-E3, is obtained from bacteria that was allowed to grow in sterile E3. We compare the bacterial secretions in E3 (i.e. BS-E3) and in ES-E3 (i.e. BS-ES) by measuring their dry weight (Fig. 5B). Next we estimated the life expectancy of the embryos incubated in same amount of secretions released by bacteria under different incubation conditions. Fig. 5C confirms that BS-E3 (bacterial secretions in E3) and BS-ES (bacterial secretions in E3 with e0, refer Fig. 4D) have significantly different effects on the embryonic life expectancy. The bacteria secretes more potent toxins (BS-ES) upon exposure to ES-E3. The comparison of TLC profile of bacterial secretions under different conditions (column1 and coulmn2) (Fig. 5D) reveals that the bacteria secretes additional compound 'b1' only if they are exposed to ES-E3 (e0). More interestingly, bacteria exposed to compounds e0-e4 (antimicrobial secretions from embryos, refer Fig 4E) alter the composition of their secretions even further as

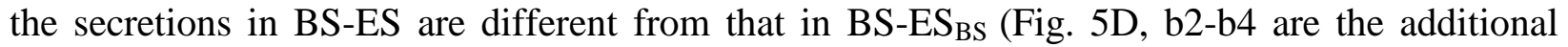
bacterial secretion compounds, Suppp. S10). Additionally, these secretions are even more potent toxins for the embryos (Supp. S11). Like embryonic secretions, the bacterial secretions are too amine containing compounds and give rise to distinct spots in Ninhydrin stained-TLC plates. Next we investigated the effect of embryonic secretions on the bacteria. Fig. 5E depicts the growth curve for bacteria (E.coli) exposed to compound e0 $\left(10^{6} / \mathrm{ml}\right.$ bacteria grown in $2 \mathrm{ml}$ ESE3) obtained from embryos having distinct $\mathrm{LDD}_{30}$ values. Significantly, higher growth rate of bacteria in ES-E3 compared to E3 indicates that the compound e0 is a nutrient for the bacteria. Thus, normal development of zebrafish embryos involves secretion of e0 which aggravates the microbial challenge for the embryo itself by accelarating the bacterial growth rate. Fig. 5F 
262 depicts the growth curve for bacteria (E.coli) exposed to e0-e4 $\left(10^{6} / \mathrm{ml}\right.$ bacterial grown in $2 \mathrm{ml}$

263 ES-BS ) obtained from embryos having distinct LDD. We observe an inverse relation between

264 the bacterial growth rate and the LDD of the embryos from which the ES-BS was obtained. This

265 observation is in agreement with Fig. 4, since the amount of antimicrobial secretion (Fig. 4B) is

266 positively correlated with $\operatorname{LDD}_{30}$ (Fig. 4A). We further validate this hypothesis by studying the

267 bacterial growth rate in ES-BS (OS). Because of lack of antimicrobial activity of ES-BS (OS),

268 the growth rate is no more dependent on LDD (Fig. 5G). 


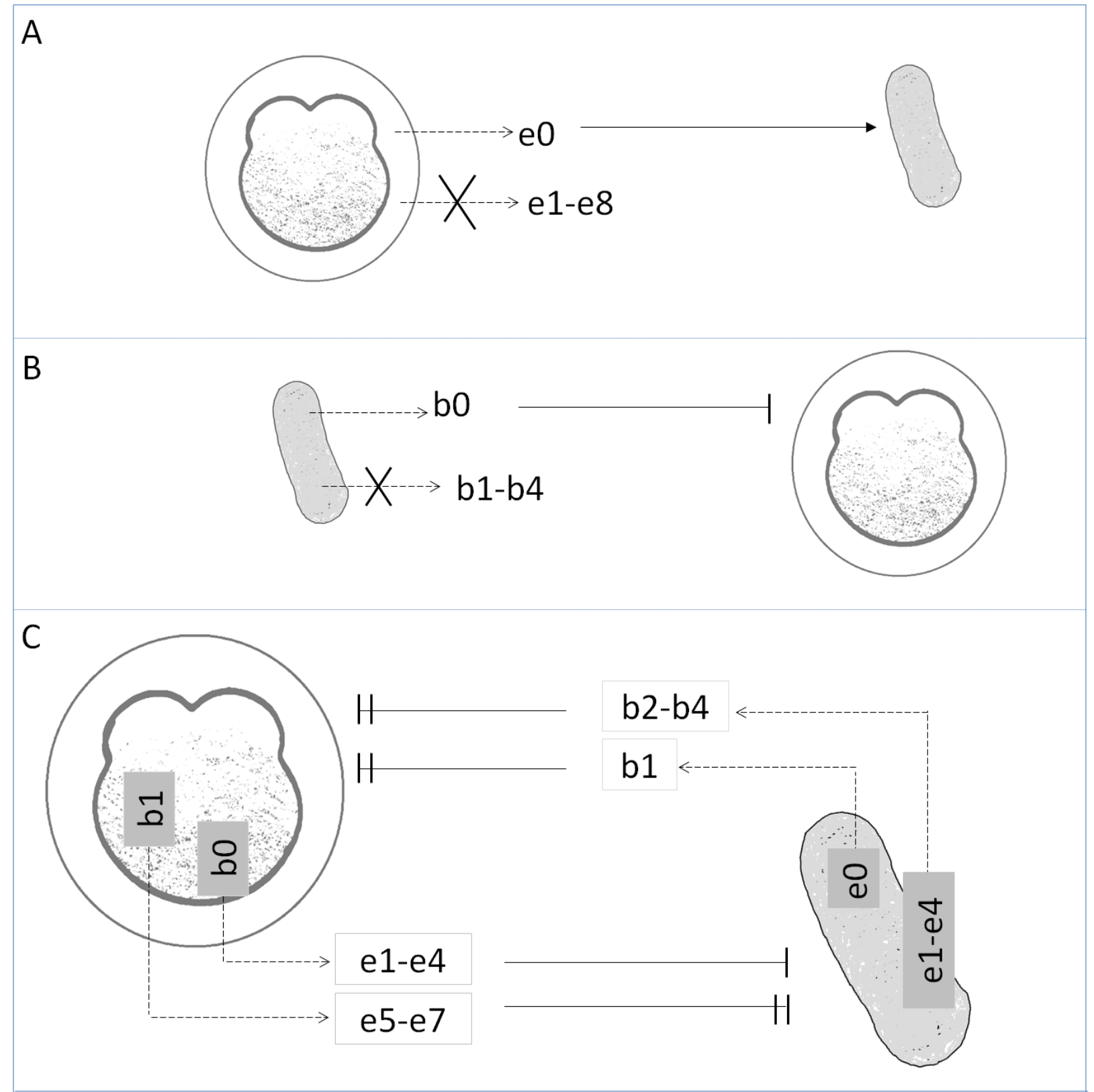

Figure 6. schamatic of the chemical warefare between the preimmune embryo and the microbes (A) secretions ( arrow with dotted line) from the embryos (e0) in microbe free medium has the ability to accelerate the growth rate of the microbes (indicated by the arrow). (B) secretions (dotted arrow) from the microbes (b0) in embryo free medium is detrimental to the development of the embryos (indicated by '--|'). (C) Response of the embryo and the microbes to microbial (b0-b4) and embryonic (e0-e8) secretions repectively. The microbe secrets b1 (more potent toxin than $\mathrm{b} 0$ ) in response to $\mathrm{e} 0$. In response to b0, the embryos secrete antimicrobial compound (e1-e4) however in response to b1 the embryo secretes additional and more potent antimicrobial compound (e5-e7). As a result the microbes secrete even more potent toxins b2-b4 in response to e1-e4. 


\section{Discussion}

281

282

283

284

285

286

287

288

289

290

291

292

293

294

295

296

297

298

299

300

301

302

Oviparous animals such as zebrafish fertilize their eggs externally. The fertilized eggs (embryos) face two types of survival challenges presented by their environment: 1) starvation and 2) pathogenic microbes. Relatively late onset of adaptive immunity in zebrafish embryos suggests their vulnerability during 0-9 hpf. Therefore, we investigated: (a) if the embryos are vulnerable to common microbes such as E.coli, and if yes, then (b) how do the embryos respond to pathogenic challenges during 0-9 hpf in the absence of any speciallized immune cells? Our observation that every clutch of embryos obtained from a given female fish has unique density of LDs (LDD) in them (Fig. 1), allows us to explore the link between LDs and survival of the embryos challenged with starvation or pathogen. LDs are synthesized on the Endoplasmic Reticulum (ER) surface. We speculate that the positive correlation in Fig. 1C arises probably from uniquely inherited ER content by the embryos. Therefore, both the $\mathrm{LDD}_{30}$ (amount of LD at $30 \mathrm{mpf}$ ) and the rate of LD synthesis are expected to be proportional to the ER surface area. By mimicking the pathogenic challenges present in the aquatic habitat (using the most ubiquitous microbe E.coli) we also show that the LDs help the embryos survive under bacteiral exposure (Fig. 2). The link between the abundunce of LDs and embryonic ability to cope with the microbes is surprising. Antimicrobial chorion of the zebrafish embryo (Fig. 3A-ii) which is the first line of defense against microbes, prevents any physical contact with microbes (Fig. 3A). The microbes attack the embryos through permeable toxic secretion (Fig. 5A). The embryos are susceptible to microbial secretions (b0, Fig. 5A) only until 9 hpf. The development of zebrafish embryos involves secretion of compound e0 (Fig. 4D) which is nutrient for bacterial growth (Fig. 5F). The embryos respond to bacterial toxin 'b0' by secreting antimicrobial amine containing compunds 'e1-e4' (Fig. 4E). Bacteria too sense the presence of embryos through embryonic 
secretion $\mathrm{e} 0$, and respond by secreting more potent pathogenic secretion 'b1' (Fig. 5B and D). Interstingly, 'e0' accelarates the bacterial growth rate (Fig. 5F). As a result more pathogenic secretion 'b0', 'b1' are generated, which compromise the survival of embryos even further. To counter the detrimental effects of pathogenic secretions 'b0', 'b1'; embryos secrete antimicrobial compounds 'e1-e4'(Fig. 5F) that checks the bacterial growth rate. The amount of embryonic secretion (e1-e4) depends on the LDD in the embryo (Fig. 4A), as a result we find a reciprocal relation between the bacterial growth rate (in ES-BS) and LDD of the embryos from which the ES-BS has been obtained (Fig. 4A). The secretion of e1-e4 by embryos is dependent on the lipolysis of the embryonic LDs (Fig. 4, 5).

We propose that both the embryos and the microbes perceive the presence of one another through the respective secretions of the other. The amount of antimicrobial compound secreted by the embryos depends on amount of LDs. To win the battle over the other, both the microbes and the embryos progressively alter their secretions to counter each other (Middle coulmn in Fig. 4E and last coulmn in Fig. 5D). This mechanism of embryonic defenses is specially functional during its pre-immune stages $(0-9 \mathrm{hpf})$. Fig. 6 is the schematic representation of the mechanism of how the embryos survive in the microbe-laced environment of their aquatic habitat.

\section{Statistical Analyses}

All statistical analyses have been performed using the 'pair-sample t-test' in OriginPro 8. The non-significant difference between compared data sets have been designated 'n.s' and signify p>0.05. The significant differences between the data sets fall under three categories: '*' depict $\mathrm{p} \leq 0.05,{ }^{\prime * * \prime}$ is for $\mathrm{p} \leq 0.01$ and ${ }^{\prime * * * '}$ denote difference between data sets at $\mathrm{p} \leq 0.001$.

\section{Experimental procedure}




\section{Zebrafish handling and embryo culture}

Wild type adult zebrafish, Danio rerio, was purchased from the local suppliers. The zebrafish were maintained at a temperature of $28^{\circ} \mathrm{C}$ and an alternate 12 hour light and 12 hour dark cycle. They were housed in a continuous water circualtion system (Aquatic Habitats Inc.). All the experiments were done in agreement with the guide lines provided by the Indian Assocition for the Cultivation fo Science, Animal Ethics Committee. Optimum care was taken at each step to reduce any pain or discomfort to the fish. For breeding, the male and female fish were tranferred to a breeding tank at a ratio of $2: 1$, respectively, and kept with separators between them overnight. Next day, the separators were removed and the fish were allowed to breed for around 20-30 min. The freshly laid embryos were collected in sterile embryo medium, E3 (50 mM NaCl, $0.17 \mathrm{mM} \mathrm{KCl}, 0.33 \mathrm{mM} \mathrm{CaCl}_{2}$ and $\left.0.33 \mathrm{mM} \mathrm{MgSO}_{4}\right)$. All the embryos were maintained in a $28^{\circ} \mathrm{C}$ incubator until used for further experimentation ${ }^{26}$.

\section{Microscopic imaging of the embryos}

\subsection{Determination of $L D D$}

'Lipid-Droplet-Density' (LDD) of the embryos was determined as described in Dutta et.al., $2015^{21}$. In brief, the embryos were embedded in $0.8 \%$ low melting agar (Puregene HiRes Agarose). The embryos were positioned with the animal pole (blastodisc) on top (top view orientation). Time-lapse images were taken at every 10 minutes interval to capture the abundance of LDs and the change in their number over time with embryonic development. Images were acquired using an upright microscope, Olympus BX61, using a water immersion 40X objective. Image processing was done using ImageJ $1.47 \mathrm{t}$ software. To determine the embryonic LDD values, we enhanced the contrast of each image first to make the LDs distinctly visible. We then counted the number of LDs visible at each time interval in the embryonic blastodisc. This was 
divided by the area of the blastodisc visible in each image and expressed as Lipid Droplet Density (LDD) per $\mathrm{mm}^{2}$ of blastodisc area. The initial value of LDD was taken as the LDD at 30 mpf $\left(\mathrm{LDD}_{30}\right.$ per $\left.\mathrm{mm}^{2}\right)$ to include the time required to prepare the sample and mounting it before imaging.

To determine the 'rate-of-increase' of LDD, the LDD values at each time point was plotted against the time points of acquiring the images (corresponding to the developmental time of the embryos) using OriginPRo 8 SR0 software. The slope of each of the plots was evaluated and plotted against the corresponding initial $\mathrm{LDD}$ value $\left(\mathrm{LDD}_{30}\right)$ for each embryo. This was done for each case and the average of at least five embryos from each clutch was plotted along with the Standard Error of Mean (S.E.M.) as the error bars.

\subsection{Staining and imaging the $L D s$}

To stain the LDs, the embryos were fixed with $4 \%$ paraformaldehyde (Merck) followed by repeated wash with Phosphate Buffer Saline (PBS). The LDs were stained using the LD-specific dye, LD540 ${ }^{27}$. Post this, LD540 was diluted to $0.1 \mathrm{~g} / \mathrm{ml}$ and the fixed embryos were soaked in it for nearly 20 minutes. The embryos were then washed two to three times with PBS followed by thourough wash with water to remove excess dye which may otherwise cause unnecessary background fluorescence. The embryos were then mounted in $0.6 \%$ low melting agar and imaged under 10X magnification using an inverted fluorescence microscope (Zeiss Axio Observer.Z1). The images were processed using the '3D-deconvolve' plugin of ImageJ $1.47 \mathrm{t}$ to minimize any unwanted background fluorescence and obtain a 3D-rendered image.

\subsection{Determining any physical contact between embryos and bacteria}

To determine whether the bacteria come in physical contact with the embryos/LDs, freshly laid embryos ( 1 cell stage) were agar-embedded and incubated with RFP-labeled bacteria $\left(10^{6} / \mathrm{ml}\right.$ 
371 E.coli DH5 $\alpha$ expressing RFP (results are with RFP-bcateria) in E3 media. The embryos were

372 oriented in lateral orientation (side view). Images were acquired by the fluorescence microscope,

373 Zeiss Axio Observer. Z1, under a $10 \mathrm{X}$ objective and green excitation light. Consecutive DIC

374 (for viewing embryo) and fluorescence (for viewing RFP-E.coli) images were acquired over time

375 along with $\mathrm{z}$-stacks at each time point. Time-lapse and z-stack images ( $5 \mu \mathrm{m}$ step size) of the

376 embryos were acquired at every 20 minutes interval. The z-stack images at each time point were

377 converted to 'maximum intensity' projections using '3D-projection' plugin of ImageJ. The

378 images were then deconvolved using '3D deconvolve' plugin of ImageJ. The 3D-rendered

379 overlaid DIC and fluorescence images at each time point give a view of the localization of the

380 bacteria and the embryo as menitoned in Fig. 4A.

3. Estimation of embryonic survival and average remaining embryonic survival expectancy

382 For comparing the survival likelihood of embryos with significantly different $\operatorname{LDD}_{30}$ values,

383 freshly laid embryos were collected from different embryo clutches. The survival of the embryos

384 under different treatment conditions was assessed every morning for the entire time of 385 observation (15 dpf in most experiments).

\subsection{Survival in the presence or absence of food supply}

387 For comparing the survival in the absence of food for embryos with different $\operatorname{LDD}_{30}$ values, 388 embryos were incubated in E3 throughout the entire duration. For the ones to be kept with food, 389 the larvae were fed with larval food from $5 \mathrm{dpf}$ (since zebrafish embryos are lecithotrophic upto $3905 \mathrm{dpf}$ ). The survival percentage of embryos was assessed at an interval of every 24 hours. 
392 To assess embryo survival in the presence or absence of bacterial exposure, freshly laid embryos

393 were collected from different clutches (different $\mathrm{LDD}_{30}$ values) and grown in the presence or

394 absence of bacteria in the medium. The survival percentage of embryos was assessed at an

395 interval of every 24 hours.

\subsection{Estimation of average remaining embryonic survival expectancy}

397 The average remaining survival expectancy of the embryos denotes the likelihood of survival of 398 the embryos under different treatment conditions. It is calculated using following formula:

$$
e_{x}=\frac{\sum_{i=x}^{15} L_{i}}{n_{x}}
$$

where, $x$ is the day of assessment,

$$
L_{x}=\frac{n_{x}+n_{x+1}}{2}
$$

All $e_{x}$ values mentioned in the manuscript denote the average remaining survival expectancy of the embryos at day zero computed over the data upto fifteen days under different experimentation conditions.

\section{Determining dry weight of secretion released by the embryos/bacteria}

\subsection{Embryonic secretions}

407 Embryos were collected at 1-cell stage in E3 medium. For yielding ES-E3 (i.e. embryonic 
413 empty tube from the weight of the tube with the dried filtrate gives the dry weight of secretion by

41430 embryos. This was used to determine weight of secretion released by 1 embryo. For obtaining

415 ES-BS, bacteria was added to E3 and allowed to grow overnight at $37^{\circ} \mathrm{C}$. The solution was

416 filtered using syringe filter (this is BS-E3) and this was used to treat 1-cell stage embryos. The

417 rest of the procudre is same as described for ES-E3. For ES-BS(OS), OS was added to BS-E3

418 and this was used to treat the embryos. Lastly, for $\mathrm{ES}-\mathrm{BS}_{\mathrm{ES}}$, bacteria was grown in previously

419 collected ES-E3 and this filtrate is termed $\mathrm{BS}_{\mathrm{ES}}$. This was used to treat the embryos. Secretions

420 released were collected in similar manner as described earlier. All experiments involving

421 determination of dry weight of secretions released by the embryos were done using 30 embryos

422 and $2 \mathrm{ml}$ of treatment solution. This were done in triplicates and the mean plotted with the

423 S.E.M. as the error bars.

\subsection{Bacterial secretions}

425 Dry weight of bacterial secretions was determined in the same procedure as the embryonic 426 secretions, except that the approximate number of bacteria yielding the dry weight was 427 determined separately and this was used to evaluate dry weight of secretion released per 428 bacterium. For this, the first step is to approximate the number of bacteria in $1 \mathrm{ml}$ culture. We 429 used RFP-E.Coli and counted the number of fluorescence dots (per bacteria) under a 10X 430 objective and green excitation light. This value was used to estimate the number of bacteria in 1 $431 \mathrm{ml}$ culture. The slope of the bacterial growth curves (Fig. 6) was multiplied with the respective 432 Optical Density (O.D.) at each time point and this was used to construct a plot of number of 433 bacteria versus time. The Boltzmann growth curve equation which was then fit into the curves is 434 as follows: 


$$
\int N(t) d t=A_{2} t+\frac{\left(A_{2}-A_{1}\right)}{k} \ln \left(e^{-k t_{0}}+e^{-k t}\right)
$$

435

where $\int N(t) d t$ is the number of bacteria at time $\mathrm{t} . \mathrm{t}=24$ hours as we evaluate bacterial secretion after 24 hours of incubation under different conditions. Upon fitting and extrapolation we deduce the different parameters. Using this we determine the secretion dry weight from each bacteria. For all experiments involving the treatment of embryos with bacterial secretions, the solution containing the secretions was evaporated in a vacuum drier and the dry weight of the secretion was evaluated. This was re-suspended in E3 to maintain a concentration of $1 \mathrm{mg} / \mathrm{ml}$ for all the treatment conditions.

\section{Drug treatment of the embryos}

\subsection{Inhibition of lipolysis by Orlistat}

The broad spectrum lipase inhibitor, Orlistat (OS) (O-STAT 120, Aristo), was used to inhibit lipolysis in the embryos. As per our previous report, the working concentration of OS i.e. 150 $\mu \mathrm{M}$ is detrimental for the development of the zebrafish embryos and causes death by $6 \mathrm{hpf}^{25}$. Hence, we determined the appropriate OS concentration at which OS treatment does not kill the embryos but delays their overall development (lipolysis still active but at a much lower rate). For this, the embryos were treated with increasing concentrations of OS (from 0 to $150 \mu \mathrm{M}$ ) and the survival of the embryos was assessed after every twenty-four hours of treatment. It is observed that at $10 \mu \mathrm{M}$ concentration of OS, the embryos survive and hence this was chosen for further experimentation so that, lipolysis is partially inhibited in these embryos, however, they do not die due to the OS treatment.

\subsection{Inhibition of protein degradation by Heclin}


455 Heclin (Tocris Bioscience) is a HECT E3 Ub ligase inhibitor ${ }^{28}$. Heclin at its working concentration $(7 \mu \mathrm{M})$ is detrimental for zebrafish embryos and kills them at around 32-cell

457 stage $^{25}$. Therefore, we determined Heclin concentration at which the embryo survive but the 458 degradation of protein is also inhibited partially. The embryos were treated with varying 459 concentrations of Heclin ( 0 to $7 \mu \mathrm{M})$ and the survival of the embryos was assessed post 24 hours 460 of incubation and the Total Protein Content (TPC) of the embryos was determined for each set. It

461 is found that Heclin at a concentration of $1 \mu \mathrm{M}$ is not lethal for the embryos but prevents the 462 degradation of protein partially as compared to the control embryos (in E3). Newly laid embryos 463 were then treated with $1 \mu \mathrm{M}$ Heclin in the presence or absence of BS-E3 and the dry weight of 464 secretion by the embryos was measured at the end of 24 hours of incubation.

\subsection{Inhibition of protein translation by Cycloheximide (CHX)}

466 Cycloheximide (Sigma) is a protein translation inhibitor. At its working concentration of $7 \mu \mathrm{M}$ it

467 is lethal to the embryos ${ }^{25}$. We varied the concentration of $\mathrm{CHX}(0$ to $1 \mu \mathrm{M})$ and determined that 468 at $1 \mu \mathrm{M} \mathrm{CHX}$ is no longer lethal to the embryos but does induce inhibition of protein translation.

469 1-cell stage embryos were treated with $1 \mu \mathrm{M} \mathrm{CHX}$ and kept with/without BS-E3 till the embryos 470 are at 16-cell stage (most secretions released within this time) (Fig. 5C). The dry weight of 471 secretion by the embryos was assessed and compared with the control embryos.

\section{6. LD isolation}

473 For LD isolation, 150 embryos were taken at 1-cell stage. The embryos were dechorionated with 474 Pronase (Sigma Aldrich) and were then deyolked using deyolking buffer (55mM NaCl, $1.8 \mathrm{mM}$ $475 \mathrm{KCl}, 1.25 \mathrm{mM} \mathrm{NaHCO} 3)^{29}<$ sup $>29</$ sup $>\mathrm{LD}$ isolation was done according to standard 476 protocol $^{30}$ with a few modifications. Post deyolking, $250 \mathrm{mM}$ of sucrose in T-buffer (300 mM 
477 glycine, $120 \mathrm{mM}$ potassium gluconate, $100 \mathrm{mM}$ HEPES, $100 \mathrm{mM}$ taurine, $20 \mathrm{mM} \mathrm{NaCl,} 2.5 \mathrm{mM}$

$478 \mathrm{MgCl}_{2}, \mathrm{pH}$ 6.8) was added to the blastodisc fraction followed by homogenization by syringe

479 plunging and pestle based homogenization. This was followed by ultracentrifugation in

480 decreasing sucrose gradient of $70 \%, 55 \%, 50 \%$ and $15 \%$ in T-buffer. LDs were collected from

481 the $15-50 \%$ layer and used for further experimentation.

\section{Disc diffusion assay using LDs, embryonic secretions}

483 An overnight culture of E. coli $(\mathrm{DH} 5 \alpha)$ was spread over agar plates using a sterilized L-spreader.

484 The plate was allowed to dry and then inverted and incubated for around $30 \mathrm{~min}$ at $37^{\circ} \mathrm{C}$ till the

485 bacterial solution had dried completely. The determination of the Anti Microbial (AM) activity

486 of the LDs and the embryonic secretions was done by the disc diffusion assay as mentioned by

487 Anand et. $\mathrm{al}^{22}$. In brief, $3 \mathrm{~mm}$ filter discs were autoclaved and placed on agar (Luria agar,

488 HiMedia). For cytostatic assay, bacteria (separate plates for gram negative, E.coli and gram

489 positive, B.subtilis bacteria) was spread on the plate and the desired sample to be tested was

490 pipetted on the discs. This was incubated overnight at $37^{\circ} \mathrm{C}$ for 24 hours. For cytotoxic assay,

491 bacteria was spread on the plate and allowed to grown overnight at $37^{\circ} \mathrm{C}$ for 24 hours. Next day,

492 the filter discs were placed and the samples were loaded on them. This was again kept for

493 overnight incubation. The clear area around the discs denotes the zone of inhibition of the

494 sample. For control, $20 \mu \mathrm{l}$ of T-buffer, $20 \mu \mathrm{l}$ of $250 \mathrm{mM}$ sucrose in T-buffer (for LD control),

$49520 \mu \mathrm{l}$ of DMSO (for LD-lipid control) and standard antibiotic, Ampicillin at the optimum

496 concentration of $50 \mu \mathrm{g} / \mathrm{ml}$ were put on the sterile discs.

\section{Acknowledgements}


The authors were financially supported by Ramanujan fellowship, funding from Department of Science and Technology and Department of Biotechnology, government of India. The work has also been supported by the institutional start up grant.

\section{Author contributions}

A.D. performed all the experiments, analyzed the data and prepared the manuscript. S.B. performed experiment, prepared the manuscript. D.K.S. conceptualized the experiments, data analysis and prepared the manuscript.

\section{References}

1. Trede, N. S. \& Zon, L. I. Development of T-cells during fish embryogenesis. Dev. Comp. Immunol. 22, 253-263 (1998).

2. Zapata, A., Diez, B., Cejalvo, T., Gutiérrez-De Frías, C. \& Cortés, A. Ontogeny of the immune system of fish. Fish Shellfish Immunol. 20, 126-136 (2006).

3. Magadan, S. Pathogen-Host Interactions: Antigenic Variation v. Somatic Adaptations. Results Probl Cell Differ. 57, 235-264 (2015).

4. Lee, J.-W. et al. RAG-1 and IgM Genes, Markers for Early Development of the Immune System in Olive Flounder, Paralichthys olivaceus. Dev. Reprod. 18, 99-106 (2014).

5. Trede, N. S., Langenau, D. M., Traver, D., Look, A. T. \& Zon, L. I. The use of zebrafish to understand immunity. Immunity 20, 367-379 (2004).

6. Lieschke, G. J. \& Currie, P. D. Animal models of human disease: Zebrafish swim into view. Nat. Rev. Genet. 8, 353-367 (2007).

7. Novoa, B. \& Figueras, A. Zebrafish: Model for the Study of Inflammation and the Innate 
8. Herbomel, P., Thisse, B. \& Thisse, C. Ontogeny and behaviour of early macrophages in the zebrafish embryo. Development 126, 3735-45 (1999).

9. Le Guyader, D. et al. Origins and unconventional behavior of neutrophils in developing zebrafisLe Guyader, Dorothée, Michael J Redd, Emma Colucci-Guyon, Emi Murayama, Karima Kissa, Valérie Briolat, Elodie Mordelet, Agustin Zapata, Hiroto Shinomiya, and Philippe Herbomel. 2007. Blood 111, 132 LP-141 (2008).

10. Herbomel, P., Thisse, B. \& Thisse, C. Zebrafish early macrophages colonize cephalic mesenchyme and developing brain, retina, and epidermis through a M-CSF receptordependent invasive process. Dev. Biol. 238, 274-288 (2001).

11. Welte, M. A. Expanding roles for lipid droplets. Curr. Biol. 25, 1-24 (2015). droplets. Nat. Rev. Mol. Cell Biol. 14, 775-786 (2013). Sci. 122, 749-752 (2009).

14. Bailey, A. P. et al. Antioxidant Role for Lipid Droplets in a Stem Cell Niche of Drosophila. Cell 163, 340-353 (2015). that Droplets Are a Protein-Storage Depot. Curr. Biol. 16, 1783-1795 (2006). 
16. Gao, Q. \& Goodman, J. M. The lipid droplet—a well-connected organelle. Front. Cell Dev. Biol. 3, 1-12 (2015).

17. den Brok, M. H., Raaijmakers, T. K., Collado-Camps, E. \& Adema, G. J. Lipid Droplets as Immune Modulators in Myeloid Cells. Trends Immunol. 39, 380-392 (2018).

18. Cubillos-Ruiz, J. R. et al. ER Stress Sensor XBP1 Controls Anti-tumor Immunity by Disrupting Dendritic Cell Homeostasis. Cell 161, 1527-1538 (2015).

19. Ibrahim, J. et al. Dendritic cell populations with different concentrations of lipid regulate tolerance and immunity in mouse and human liver. Gastroenterology 143, 1061-1072 (2012).

20. Wang, S., Wang, Y., Ma, J., Ding, Y. \& Zhang, S. Phosvitin plays a critical role in the immunity of zebrafish embryos via acting as a pattern recognition receptor and an antimicrobial effector. J. Biol. Chem. 286, 22653-22664 (2011).

21. Dutta, A. \& Kumar Sinha, D. Turnover of the actomyosin complex in zebrafish embryos directs geometric remodelling and the recruitment of lipid droplets. Sci. Rep. 5, 1-14 (2015).

22. Anand, P. et al. A novel role for lipid droplets in the organismal antibacterial response. Elife 2012, 1-18 (2012).

23. Heck, A. M., Yanovski, J. A. \& Calis, K. A. Orlistat, a new lipase inhibitor for the management of obesity. Pharmacotherapy 20, 270-279 (2000).

24. Sternby, B., Hartmann, D., Borgström, B. \& Nilsson, Å. Degree of in vivo inhibition of human gastric and pancreatic lipases by Orlistat (Tetrahydrolipstatin, THL) in the stomach 
and small intestine. Clin. Nutr. 21, 395-402 (2002).

561 25. Dutta, A. \& Sinha, D. K. Zebrafish lipid droplets regulate embryonic ATP homeostasis to 562 power early development. Open Biol. 7, (2017).

563 26. Avdesh, A. et al. Regular Care and Maintenance of a Zebrafish (Danio rerio) Laboratory: An Introduction. J. Vis. Exp. (2012). doi:10.3791/4196

565 27. Spandl, J., White, D. J., Peychl, J. \& Thiele, C. Live cell multicolor imaging of lipid 566 droplets with a new dye, LD540. Traffic 10, 1579-1584 (2009).

28. Mund, T., Lewis, M. J., Maslen, S. \& Pelham, H. R. Peptide and small molecule inhibitors of HECT-type ubiquitin ligases. Proc. Natl. Acad. Sci. 111, 16736-16741 (2014). BMC Dev. Biol. 6, 1 (2006). 